\title{
A Two-parameter Balakrishnan Skew-normal Distribution
}

\author{
Wahab Bahrami, ${ }^{\dagger}, *$ Hamzeh Agahi ${ }^{\dagger}$ and Hojat Rangin ${ }^{\ddagger}$ \\ † Shahid Bahonar University of Kerman \\ $\ddagger$ Tarbiat Moalem University of Tehran
}

\begin{abstract}
In this paper, we discuss a generalization of Balakrishnan skewnormal distribution with two parameters that contains the skew-normal, the Balakrishnan skew-normal and the two-parameter generalized skew-normal distributions as special cases. Furthermore, we establish some useful properties and two extensions of this distribution.
\end{abstract}

Keywords. Skew-normal distribution; Balakrishnan skew-normal distribution; orthant probability; moment generating function.

\section{Introduction}

Azzalini (1985) introduced the skew-normal class as one being able to reflect varying degrees of skewnees, which is mathematically tractable and which includes the normal distribution as a special case.

A random variable $Z$ has a standard skew-normal distribution with parameter $\lambda \in \mathbb{R}$, denoted by $Z \sim S N(\lambda)$, if its pdf is

$$
f(z ; \lambda)=2 \varphi(z) \Phi(\lambda z) \quad z \in \mathbb{R}
$$

where $\varphi(\cdot)$ and $\Phi(\cdot)$ are the pdf and cdf of the standard normal distribution, respectively. This distribution and its generalizations have been studied by some researchers. For example, Arellano-Valle et al. (2004), Arnold and Beaver (2002), Azzalini (1986), Azzalini and Dalla valle (1996), Branco and

* Corresponding author 
Dey (2001) and Henze (1986). Balakrishnan (2002), in discussion, proposed a generalization of standard skew-normal distribution as follows:

A random variable $Z$ has a Balakrishnan skew-normal distribution with parameter $\lambda \in \mathbb{R}$, denoted by $Z \sim B S N_{n}(\lambda)$, if its pdf is

$$
f_{n}(z ; \lambda)=\frac{1}{c_{n}(\lambda)} \varphi(z)[\Phi(\lambda z)]^{n} \quad z \in \mathbb{R},
$$

where $n$ is a non-negative integer number and if $V, U_{1}, \ldots, U_{n} \stackrel{i i d}{\sim} N(0,1)$, then $c_{n}(\lambda)$ is given by

$$
c_{n}(\lambda)=\int_{-\infty}^{+\infty} \varphi(z)[\Phi(\lambda z)]^{n} d z=E\left[\Phi^{n}(\lambda V)\right]=\operatorname{Pr}\left(U_{1} \leqslant \lambda V, \ldots, U_{n} \leqslant \lambda V\right) .
$$

In special cases (see Gupta and Gupta, 2004, or Jamalzadeh et al., 2008), by using the orthant probability expression, we have

$$
c_{2}(\lambda)=\frac{1}{\pi} \tan ^{-1}\left(\sqrt{1+2 \lambda^{2}}\right) .
$$

This distribution has been studied and generalized by several authors, for example, Gupta and Gupta (2004), Sharafi and Behboodian (2008). Recently, Yadegari et al. (2008) introduced a new generalization of the Balakrishnan skew-normal distribution by explaining some important properties of this distribution. They introduced the generalized distribution as follows:

A random variable $Z$ has a generalized Balakrishnan skew-normal distribution with parameter $\lambda \in \mathbb{R}$, denoted by $G B S N_{n, m}(\lambda)$, if its density is

$$
f_{n, m}(z ; \lambda)=\frac{1}{c_{n, m}(\lambda)}[\Phi(\lambda z)]^{n}[1-\Phi(\lambda z)]^{m} \varphi(z) \quad z \in \mathbb{R},
$$

where $c_{n, m}(\lambda)=\sum_{i=0}^{m}\left(\begin{array}{c}m \\ i\end{array}\right)(-1)^{i} \int_{-\infty}^{+\infty}[\Phi(\lambda z)]^{n+i} \varphi(z) d z$, and $n, m$ are nonnegative integer numbers.

Also, Jamalizadeh et al. (2008) introduced a new class of two parameter skew-normal distribution as follows:

A random variable $Z$ has a two-parameter skew-normal distribution with parameters $\lambda_{1}, \lambda_{2} \in \mathbb{R}$, denoted by $G S N\left(\lambda_{1}, \lambda_{2}\right)$, if its pdf is

$$
f\left(z ; \lambda_{1}, \lambda_{2}\right)=\frac{2 \pi}{\cos ^{-1}\left(\frac{-\lambda_{1} \lambda_{2}}{\sqrt{1+\lambda_{1}^{2}} \sqrt{1+\lambda_{2}^{2}}}\right)} \varphi(z) \Phi\left(\lambda_{1} z\right) \Phi\left(\lambda_{2} z\right) \quad z \in \mathbb{R} .
$$


For the special case of this density function, if $\lambda_{1}=\lambda_{2}=\lambda$, then $G S N(\lambda, \lambda)=B S N_{2}(\lambda)$. In this paper we introduce a new distribution named a two-parameter Balakrishnan skew-normal distribution, denoted by $T B S N_{n, m}\left(\lambda_{1}, \lambda_{2}\right) ; S N(\lambda), B S N_{n}(\lambda), G B S N_{n, m}(\lambda)$, and $G S N\left(\lambda_{1}, \lambda_{2}\right)$ are special cases of this distribution.

The paper is arranged as follows. In the next section, we present the definition and some simple properties of $T B S N_{n, m}\left(\lambda_{1}, \lambda_{2}\right)$. In Section 3 , some important theorems concerning several useful properties are given. The moment generating function and some important theorems about the moments of this distribution are derived in Section 4. Finally, two extensions of this distribution are introduced.

\section{A Two-parameter Balakrishnan Skew-normal Dis- tribution}

In this section, we present the definition and some simple properties of $T B S N_{n, m}\left(\lambda_{1}, \lambda_{2}\right)$.

Definition 1 A random variable $Z$ has a two-parameter Balakrishnan skewnormal distribution with parameters $\lambda_{1}, \lambda_{2} \in \mathbb{R}$, denoted by $T B S N_{n, m}\left(\lambda_{1}, \lambda_{2}\right)$, if its pdf is

$$
f_{n, m}\left(z ; \lambda_{1}, \lambda_{2}\right)=\frac{1}{c_{n, m}\left(\lambda_{1}, \lambda_{2}\right)} \varphi(z)\left[\Phi\left(\lambda_{1} z\right)\right]^{n}\left[\Phi\left(\lambda_{2} z\right)\right]^{m} \quad z \in \mathbb{R}
$$

where $c_{n, m}\left(\lambda_{1}, \lambda_{2}\right)=E\left\{\left[\Phi\left(\lambda_{1} X\right)\right]^{n}\left[\Phi\left(\lambda_{2} X\right)\right]^{m}\right\}, X \sim N(0,1)$, and $n, m$ are non-negative integer numbers.

Now, we show how $c_{n, m}\left(\lambda_{1}, \lambda_{2}\right)$ can be calculated by orthant probability. In fact an orthant probability is the probability $P\left(X_{1}>0, \ldots, X_{n}>0\right)$ with $X_{1}, \ldots, X_{n} \sim N_{n}(\mathbf{0}, \Sigma)$, where $\Sigma$ is an $n \times n$ diagonal matrix with diagonal elements 1 and non-diagonal elements $\rho_{i j}$ (See, for example, Kotz and Johnson, 1985, and Kots et al., 2000).

If $X, V_{1}, \ldots, V_{n}, U_{1}, \ldots, U_{m} \stackrel{i i d}{\sim} N(0,1)$, then we have

$$
\left(\frac{\lambda_{1} X-V_{1}}{\sqrt{1+\lambda_{1}^{2}}}, \ldots, \frac{\lambda_{1} X-V_{n}}{\sqrt{1+\lambda_{1}^{2}}}, \frac{\lambda_{2} X-U_{1}}{\sqrt{1+\lambda_{2}^{2}}}, \ldots, \frac{\lambda_{2} X-U_{m}}{\sqrt{1+\lambda_{2}^{2}}}\right) \sim N_{n+m}\left(\mathbf{0},\left(\begin{array}{cc}
\Sigma & \Lambda \\
\Lambda^{T} & \Omega
\end{array}\right)\right)
$$


where $\Sigma$ is an $n \times n$ diagonal matrix with diagonal elements 1 and nondiagonal elements $\frac{\lambda_{1}^{2}}{1+\lambda_{1}^{2}}, \Omega$ is an $m \times m$ diagonal matrix with diagonal elements 1 and non-diagonal elements $\frac{\lambda_{2}^{2}}{1+\lambda_{2}^{2}}$, and $\Lambda$ is an $n \times m$ matrix whose all elements are $\frac{\lambda_{1} \lambda_{2}}{\sqrt{1+\lambda_{1}^{2}} \sqrt{1+\lambda_{2}^{2}}}$. Then

$$
\begin{aligned}
c_{n, m}\left(\lambda_{1}, \lambda_{2}\right)= & E\left\{\left[\Phi\left(\lambda_{1} X\right)\right]^{n}\left[\Phi\left(\lambda_{2} X\right)\right]^{m}\right\} \\
= & \operatorname{Pr}\left(\frac{\lambda_{1} X-V_{1}}{\sqrt{1+\lambda_{1}^{2}}}>0, \ldots, \frac{\lambda_{1} X-V_{n}}{\sqrt{1+\lambda_{1}^{2}}}>0, \frac{\lambda_{2} X-U_{1}}{\sqrt{1+\lambda_{2}^{2}}}>0,\right. \\
& \left.\ldots, \frac{\lambda_{2} X-U_{m}}{\sqrt{1+\lambda_{2}^{2}}}>0\right) .
\end{aligned}
$$

Therefore, $c_{n, m}\left(\lambda_{1}, \lambda_{2}\right)$ is an orthant probability. For large $n, m$ there is no closed form for $c_{n, m}\left(\lambda_{1}, \lambda_{2}\right)$ but one can find some approximate values (see Steck, 1962, and Kotz and Johnson, 1985).

We cite some simple properties of $T B S N_{n, m}\left(\lambda_{1}, \lambda_{2}\right)$ in the next theorem.

Theorem 1. Let $Z \sim T B S N_{n, m}\left(\lambda_{1}, \lambda_{2}\right)$. Then,

(1) $T B S N_{1,1}\left(\lambda_{1}, 0\right)=S N\left(\lambda_{1}\right)$ and $T B S N_{1,1}\left(0, \lambda_{2}\right)=S N\left(\lambda_{2}\right)$;

(2) $T B S N_{n, m}(\lambda, \lambda)=B S N_{n+m}(\lambda)$;

(3) $T B S N_{n, m}\left(\lambda_{1}, 0\right)=B S N_{n}\left(\lambda_{1}\right)$ and $T B S N_{n, m}\left(0, \lambda_{2}\right)=B S N_{m}\left(\lambda_{2}\right)$;

(4) $\operatorname{TBSN_{1,1}}\left(\lambda_{1}, \lambda_{2}\right)=G S N\left(\lambda_{1}, \lambda_{2}\right)$;

(5) $T B S N_{n, m}(\lambda,-\lambda)=G B S N_{n, m}(\lambda)$ and $T B S N_{n, m}(-\lambda, \lambda)=G B S N_{m, n}(\lambda)$;

(6) $T B S N_{n, m}(0,0)=T B S N_{0,0}\left(\lambda_{1}, \lambda_{2}\right)=N(0,1)$;

(7) $-Z \sim T B S N_{n, m}\left(-\lambda_{1},-\lambda_{2}\right)$;

(8) Let $Z_{1}, \ldots, Z_{n} \stackrel{\text { iid }}{\sim} N(0,1)$ and $Z_{1: n} \leqslant Z_{2: n} \leqslant \cdots \leqslant Z_{n: n}$ be the corresponding order statistics, then $Z_{i: n} \sim T B S N_{i-1, n-i}(1,-1)$.

Proof. The proof is simple.

Figure 1 shows the shape of $T B S N_{n, m}\left(\lambda_{1}, \lambda_{2}\right)$ for some values of $n, m$, $\lambda_{1}$, and $\lambda_{2}$. 


\section{Some Important Properties of $T B S N_{n, m}\left(\lambda_{1}, \lambda_{2}\right)$}

In this Section, we derive the some properties of $T B S N_{n, m}\left(\lambda_{1}, \lambda_{2}\right)$ distribution.

Theorem 2. (Representation Theorem) If $X, V_{1}, \ldots, V_{n}, U_{1}, \ldots, U_{m} \stackrel{i i d}{\sim} N(0,1)$, $V_{n: n}=\max \left(V_{1}, \ldots, V_{n}\right)$, and $U_{m: m}=\max \left(U_{1}, \ldots, U_{m}\right)$, then

$$
X \mid\left(V_{n: n}<\lambda_{1} X, U_{m: m}<\lambda_{2} X\right) \sim T B S N_{n, m}\left(\lambda_{1}, \lambda_{2}\right) .
$$

Proof. If $W=X \mid\left(V_{n: n}<\lambda_{1} X, U_{m: m}<\lambda_{2} X\right)$, then we have

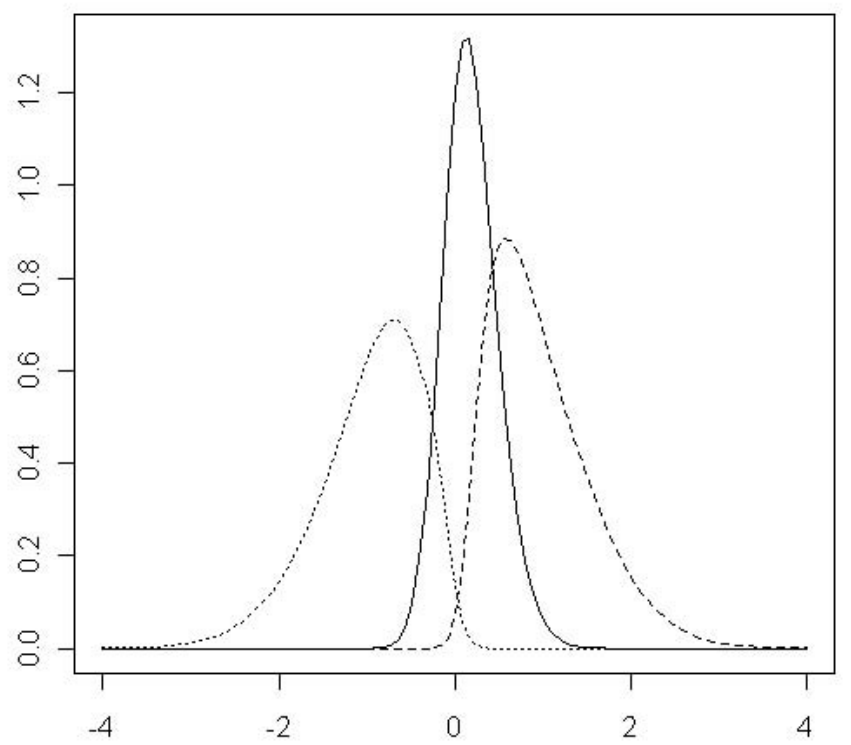

Figure 1. Some shapes of $T B S N_{n, m}\left(\lambda_{1}, \lambda_{2}\right): T B S N_{1,1}(-2,4)$ (solid line), $T B S N_{2,1}(-2,7)$ (dotted line), $T B S N_{2,2}(3,5)$ (dashed line). 


$$
\begin{aligned}
f_{W}(x) & =\frac{\operatorname{Pr}\left(V_{n: n}<\lambda_{1} X, U_{m: m}<\lambda_{2} X \mid X=x\right) \varphi(x)}{\operatorname{Pr}\left(V_{n: n}<\lambda_{1} X, U_{m: m}<\lambda_{2} X\right)} \\
& =\frac{\operatorname{Pr}\left(V_{n: n}<\lambda_{1} x, U_{m: m}<\lambda_{2} x\right) \varphi(x)}{\operatorname{Pr}\left(V_{n: n}<\lambda_{1} X, U_{m: m}<\lambda_{2} X\right)} \\
& =\frac{\left[\Phi\left(\lambda_{1} x\right)\right]^{n}\left[\Phi\left(\lambda_{2} x\right)\right]^{m} \varphi(x)}{\operatorname{Pr}\left(V_{n: n}<\lambda_{1} X, U_{m: m}<\lambda_{2} X\right)} .
\end{aligned}
$$

Also

$$
\begin{aligned}
\operatorname{Pr}\left(V_{n: n}<\lambda_{1} X, U_{m: m}<\lambda_{2} X\right)= & \int_{-\infty}^{+\infty} \operatorname{Pr}\left(V_{n: n}<\lambda_{1} x, U_{m: m}<\lambda_{2} x\right) \varphi(x) d x \\
= & \int_{-\infty}^{+\infty} \operatorname{Pr}\left(V_{1}<\lambda_{1} x, \ldots, V_{n}<\lambda_{1} x, U_{1}<\lambda_{2} x,\right. \\
& \left.\ldots, U_{m}<\lambda_{2} x\right) \varphi(x) d x \\
= & \int_{-\infty}^{+\infty}\left[\Phi\left(\lambda_{1} x\right)\right]^{n}\left[\Phi\left(\lambda_{2} x\right)\right]^{m} \varphi(x) d x \\
= & c_{n, m}\left(\lambda_{1}, \lambda_{2}\right) .
\end{aligned}
$$

Therefore, $X \mid\left(V_{n: n}<\lambda_{1} X, U_{m: m}<\lambda_{2} X\right) \sim T B S N_{n, m}\left(\lambda_{1}, \lambda_{2}\right)$ and the proof is completed.

Theorem 3. The density $f_{n, m}\left(z ; \lambda_{1}, \lambda_{2}\right)$ introduced in Definition 1, is strongly unimodal.

Proof. To prove that $f_{n, m}\left(z ; \lambda_{1}, \lambda_{2}\right)$ is strongly unimodal, it is enough to show that $f_{n, m}\left(z ; \lambda_{1}, \lambda_{2}\right)$ is a logconcave function of $z$, for all $z \in \mathbb{R}$. Thus we have

$$
\begin{aligned}
\frac{d^{2}}{d z^{2}} \log f_{n, m}\left(z ; \lambda_{1}, \lambda_{2}\right)= & -n \lambda_{1}^{2} \frac{\varphi\left(\lambda_{1} z\right)}{\Phi\left(\lambda_{1} z\right)}\left[\lambda_{1} z+\frac{\varphi\left(\lambda_{1} z\right)}{\Phi\left(\lambda_{1} z\right)}\right] \\
& -m \lambda_{2}^{2} \frac{\varphi\left(\lambda_{2} z\right)}{\Phi\left(\lambda_{2} z\right)}\left[\lambda_{2} z+\frac{\varphi\left(\lambda_{2} z\right)}{\Phi\left(\lambda_{2} z\right)}\right]-1<0 .
\end{aligned}
$$

Since $\varphi(t)+t \Phi(t)>0$, for all $t \in \mathbb{R}$ (see Azzalini, 1986) then the above expression is negative.

Theorem 4. If $Z \sim T B S N_{n-1, m-1}\left(\lambda_{1}, \lambda_{2}\right)$ and $Y_{1}, Y_{2} \stackrel{i i d}{\sim} N(0,1)$ are independent, then

$$
Z \mid\left(Y_{1}<\lambda_{1} Z, Y_{2}<\lambda_{2} Z\right) \sim T B S N_{n, m}\left(\lambda_{1}, \lambda_{2}\right) .
$$


Proof. If $X=Z \mid\left(Y_{1}<\lambda_{1} Z, Y_{2}<\lambda_{2} Z\right)$, then we have the followings

$$
\begin{gathered}
\operatorname{Pr}(X \leqslant x)=\frac{\operatorname{Pr}\left(Z \leqslant x, Y_{1}<\lambda_{1} Z, Y_{2}<\lambda_{2} Z\right)}{\operatorname{Pr}\left(Y_{1}<\lambda_{1} Z, Y_{2}<\lambda_{2} Z\right)}, \\
\operatorname{Pr}\left(Z \leqslant x, Y_{1}<\lambda_{1} Z, Y_{2}<\lambda_{2} Z\right)=\int_{-\infty}^{x} \frac{1}{c_{n-1, m-1}\left(\lambda_{1}, \lambda_{2}\right)} \\
\varphi(z)\left[\Phi\left(\lambda_{1} z\right)\right]^{n}\left[\Phi\left(\lambda_{2} z\right)\right]^{m} d z,
\end{gathered}
$$

and

$$
\begin{aligned}
\operatorname{Pr}\left(Y_{1}<\lambda_{1} Z, Y_{2}<\lambda_{2} Z\right) & =\int_{-\infty}^{+\infty} \frac{1}{c_{n-1, m-1}\left(\lambda_{1}, \lambda_{2}\right)} \varphi(z)\left[\Phi\left(\lambda_{1} z\right)\right]^{n}\left[\Phi\left(\lambda_{2} z\right)\right]^{m} d z \\
& =\frac{c_{n, m}\left(\lambda_{1}, \lambda_{2}\right)}{c_{n-1, m-1}\left(\lambda_{1}, \lambda_{2}\right)} .
\end{aligned}
$$

Therefore,

$$
\operatorname{Pr}(X \leqslant x)=\int_{-\infty}^{x} \frac{1}{c_{n, m}\left(\lambda_{1}, \lambda_{2}\right)} \varphi(z)\left[\Phi\left(\lambda_{1} z\right)\right]^{n}\left[\Phi\left(\lambda_{2} z\right)\right]^{m} d z .
$$

By taking derivative from the above expression with respect to $x$, we have

$$
f_{n, m}\left(x ; \lambda_{1}, \lambda_{2}\right)=\frac{1}{c_{n, m}\left(\lambda_{1}, \lambda_{2}\right)} \varphi(x)\left[\Phi\left(\lambda_{1} x\right)\right]^{n}\left[\Phi\left(\lambda_{2} x\right)\right]^{m}
$$

and the proof is completed.

\section{Moments of $T B S N_{n, m}\left(\lambda_{1}, \lambda_{2}\right)$}

In this section, we give the moment generating function (mgf) of $Z \sim$ $T B S N_{n, m}\left(\lambda_{1}, \lambda_{2}\right)$. The mgf of $Z^{2}$ has a closed form for even moments of $T B S N_{n, m}\left(\lambda_{1}, \lambda_{2}\right)$. Furthermore, we present $E\left(Z^{k}\right)$ for any $k \in N$.

Theorem 5. The moment generating function of $Z \sim T B S N_{n, m}\left(\lambda_{1}, \lambda_{2}\right)$ is

$$
M_{Z}(t)=\frac{e^{\frac{t^{2}}{2}}}{c_{n, m}\left(\lambda_{1}, \lambda_{2}\right)} E_{X}\left\{\left[\Phi\left(\lambda_{1}(X+t)\right)\right]^{n}\left[\Phi\left(\lambda_{2}(X+t)\right)\right]^{m}\right\},
$$

where $X \sim N(0,1)$. 
Proof. The proof is simple.

Theorem 6. Let $k \in N$. If $Z \sim T B S N_{n, m}\left(\lambda_{1}, \lambda_{2}\right)$, then

$$
\begin{aligned}
E\left(Z^{k}\right)= & (k-1) E\left(Z^{k-2}\right)+\frac{n \lambda_{1}\left(1+\lambda_{1}^{2}\right)^{-\frac{k}{2}}}{\sqrt{2 \pi}} \cdot \frac{c_{n-1, m}\left(\frac{\lambda_{1}}{\sqrt{1+\lambda_{1}^{2}}}, \frac{\lambda_{2}}{\sqrt{1+\lambda_{1}^{2}}}\right)}{c_{n, m}\left(\lambda_{1}, \lambda_{2}\right)} \\
& \times E\left(U^{k-1}\right)+\frac{m \lambda_{2}\left(1+\lambda_{2}^{2}\right)^{-\frac{k}{2}}}{\sqrt{2 \pi}} \cdot \frac{c_{n, m-1}\left(\frac{\lambda_{1}}{\sqrt{1+\lambda_{2}^{2}}}, \frac{\lambda_{2}}{\sqrt{1+\lambda_{2}^{2}}}\right)}{c_{n, m}\left(\lambda_{1}, \lambda_{2}\right)} E\left(V^{k-1}\right),
\end{aligned}
$$

where

$$
U \sim T B S N_{n-1, m}\left(\frac{\lambda_{1}}{\sqrt{1+\lambda_{1}^{2}}}, \frac{\lambda_{2}}{\sqrt{1+\lambda_{1}^{2}}}\right)
$$

and

$$
V \sim T B S N_{n, m-1}\left(\frac{\lambda_{1}}{\sqrt{1+\lambda_{2}^{2}}}, \frac{\lambda_{2}}{\sqrt{1+\lambda_{2}^{2}}}\right)
$$

are independent.

\section{Proof.}

$$
\begin{aligned}
E\left(Z^{k}\right)= & \int_{-\infty}^{+\infty} \frac{1}{c_{n, m}\left(\lambda_{1}, \lambda_{2}\right)} z^{k-1} z \varphi(z)\left[\Phi\left(\lambda_{1} z\right)\right]^{n}\left[\Phi\left(\lambda_{2} z\right)\right]^{m} d z \\
= & \frac{(k-1)}{c_{n, m}\left(\lambda_{1}, \lambda_{2}\right)} \int_{-\infty}^{+\infty} z^{k-2} \varphi(z)\left[\Phi\left(\lambda_{1} z\right)\right]^{n}\left[\Phi\left(\lambda_{2} z\right)\right]^{m} \\
& +\frac{n \lambda_{1}}{c_{n, m}\left(\lambda_{1}, \lambda_{2}\right)} \int_{-\infty}^{+\infty} z^{k-1} \varphi(z) \varphi\left(\lambda_{1} z\right)\left[\Phi\left(\lambda_{1} z\right)\right]^{n-1}\left[\Phi\left(\lambda_{2} z\right)\right]^{m} d z \\
& +\frac{m \lambda_{2}}{c_{n, m}\left(\lambda_{1}, \lambda_{2}\right)} \int_{-\infty}^{+\infty} z^{k-1} \varphi(z) \varphi\left(\lambda_{2} z\right)\left[\Phi\left(\lambda_{1} z\right)\right]^{n}\left[\Phi\left(\lambda_{2} z\right)\right]^{m-1} d z
\end{aligned}
$$




$$
\begin{aligned}
= & \frac{(k-1)}{c_{n, m}\left(\lambda_{1}, \lambda_{2}\right)} \int_{-\infty}^{+\infty} z^{k-2} \varphi(z)\left[\Phi\left(\lambda_{1} z\right)\right]^{n}\left[\Phi\left(\lambda_{2} z\right)\right]^{m} \\
& +\frac{n \lambda_{1}\left(1+\lambda_{1}^{2}\right)^{-\frac{k}{2}}}{c_{n, m}\left(\lambda_{1}, \lambda_{2}\right) \sqrt{2 \pi}} \int_{-\infty}^{+\infty} x^{k-1} \varphi(x)\left[\Phi\left(\frac{\lambda_{1}}{\sqrt{1+\lambda_{1}^{2}}} x\right)\right]^{n-1} \\
& \times\left[\Phi\left(\frac{\lambda_{2}}{\sqrt{1+\lambda_{1}^{2}}} x\right)\right]^{m} d x \\
= & (k-1) E\left(Z^{k-2}\right)+\frac{n \lambda_{1}\left(1+\lambda_{1}^{2}\right)^{-\frac{k}{2}}}{\sqrt{2 \pi}} \cdot \frac{c_{n-1, m}\left(\frac{\lambda_{1}}{\sqrt{1+\lambda_{1}^{2}}}, \frac{\lambda_{2}}{\sqrt{1+\lambda_{1}^{2}}}\right)}{c_{n, m}\left(\lambda_{1}, \lambda_{2}\right)} \\
& \times E\left(U^{k-1}\right)+\frac{m \lambda_{2}\left(1+\lambda_{2}^{2}\right)^{-\frac{k}{2}}}{\sqrt{2 \pi}} \cdot \frac{c_{n, m-1}\left(\frac{\lambda_{1}}{\sqrt{1+\lambda_{2}^{2}}}, \frac{\lambda_{2}}{\sqrt{1+\lambda_{2}^{2}}}\right)}{c_{n, m}\left(\lambda_{1}, \lambda_{2}\right)} E\left(V^{k-1}\right) .
\end{aligned}
$$

Corollary 1 If $Z \sim T B S N_{n, m}\left(\lambda_{1}, \lambda_{2}\right)$, then

$$
\begin{aligned}
E(Z)= & \frac{n \lambda_{1}}{\sqrt{2 \pi\left(1+\lambda_{1}^{2}\right)}} \cdot \frac{c_{n-1, m}\left(\frac{\lambda_{1}}{\sqrt{1+\lambda_{1}^{2}}}, \frac{\lambda_{2}}{\sqrt{1+\lambda_{1}^{2}}}\right)}{c_{n, m}\left(\lambda_{1}, \lambda_{2}\right)} \\
& +\frac{m \lambda_{2}}{\sqrt{2 \pi\left(1+\lambda_{2}^{2}\right)}} \cdot \frac{c_{n, m-1}\left(\frac{\lambda_{1}}{\sqrt{1+\lambda_{2}^{2}}}, \frac{\lambda_{2}}{\sqrt{1+\lambda_{2}^{2}}}\right)}{c_{n, m}\left(\lambda_{1}, \lambda_{2}\right)} .
\end{aligned}
$$

Theorem 7. If $Z \sim T B S N_{n, m}\left(\lambda_{1}, \lambda_{2}\right)$, then

$$
M_{Z^{2}}(t)=(1-2 t)^{-\frac{1}{2}} \cdot \frac{c_{n, m}\left(\frac{\lambda_{1}}{\sqrt{1-2 t}}, \frac{\lambda_{2}}{\sqrt{1-2 t}}\right)}{c_{n, m}\left(\lambda_{1}, \lambda_{2}\right)} .
$$




\section{Proof.}

$$
\begin{aligned}
M_{Z^{2}}(t) & =E\left(e^{t Z^{2}}\right)=\frac{1}{c_{n, m}\left(\lambda_{1}, \lambda_{2}\right)} \int_{-\infty}^{+\infty} e^{t z^{2}} \varphi(z)\left[\Phi\left(\lambda_{1} z\right)\right]^{n}\left[\Phi\left(\lambda_{2} z\right)\right]^{m} d z \\
& =\frac{1}{c_{n, m}\left(\lambda_{1}, \lambda_{2}\right)} \int_{-\infty}^{+\infty} \frac{1}{\sqrt{2 \pi}} e^{-\frac{1}{2}(1-2 t) z^{2}}\left[\Phi\left(\lambda_{1} z\right)\right]^{n}\left[\Phi\left(\lambda_{2} z\right)\right]^{m} d z \\
& =\frac{(1-2 t)^{-\frac{1}{2}}}{c_{n, m}\left(\lambda_{1}, \lambda_{2}\right)} \int_{-\infty}^{+\infty} \varphi(x)\left[\Phi\left(\frac{\lambda_{1}}{\sqrt{1-2 t}} x\right)\right]^{n}\left[\Phi\left(\frac{\lambda_{2}}{\sqrt{1-2 t}} x\right)\right]^{m} d x \\
& =(1-2 t)^{-\frac{1}{2}} \frac{c_{n, m}\left(\frac{\lambda_{1}}{\sqrt{1-2 t}}, \frac{\lambda_{2}}{\sqrt{1-2 t}}\right)}{c_{n, m}\left(\lambda_{1}, \lambda_{2}\right)} .
\end{aligned}
$$

By Theorem 7, we can calculate the even moments of $T B S N_{n, m}\left(\lambda_{1}, \lambda_{2}\right)$.

\section{Some Extensions of $T B S N_{n, m}\left(\lambda_{1}, \lambda_{2}\right)$}

In this section, we introduce two extensions of $T B S N_{n, m}\left(\lambda_{1}, \lambda_{2}\right)$.

Definition 2 A location-scale two-parameter Balakrishnan skew-normal distribution is defined as that of $X=\mu+\sigma Z$, where $Z \sim T B S N_{n, m}\left(\lambda_{1}, \lambda_{2}\right)$, $\mu \in \mathbb{R}$ and $\sigma>0$. Its density is given by

$$
\begin{aligned}
f_{n, m}(x ; \boldsymbol{\theta})= & \frac{1}{\sigma c_{n, m}\left(\lambda_{1}, \lambda_{2}\right)} \varphi\left(\frac{x-\mu}{\sigma}\right)\left[\Phi\left(\lambda_{1} \frac{x-\mu}{\sigma}\right)\right]^{n} \\
& \times\left[\Phi\left(\lambda_{2} \frac{x-\mu}{\sigma}\right)\right]^{m} \quad x \in \mathbb{R} .
\end{aligned}
$$

Definition 3 A random vector $\mathbf{Z}$ has a multivariate two vector parameters Balakrishnan skew-normal distribution with $\boldsymbol{\alpha}, \boldsymbol{\beta}$ vector parameters, denoted by $T B S N_{n, m}^{k}\left(\boldsymbol{\lambda}_{1}, \boldsymbol{\lambda}_{2}\right)$, if its pdf is

$$
f_{n, m}(\mathbf{z} ; \boldsymbol{\alpha}, \boldsymbol{\beta}, \Omega)=\frac{1}{c} \varphi_{k}(\mathbf{z}, \Omega)\left[\Phi\left(\boldsymbol{\alpha}^{T} \mathbf{z}\right)\right]^{n}\left[\Phi\left(\boldsymbol{\beta}^{T} \mathbf{z}\right)\right]^{m} \quad \mathbf{z} \in \mathbb{R}^{k}
$$

where $\mathbf{z}^{T}=\left(z_{1}, \ldots, z_{k}\right)^{T}, \boldsymbol{\alpha}^{T}=\left(\alpha_{1}, \ldots, \alpha_{k}\right)^{T}, \boldsymbol{\beta}^{T}=\left(\beta_{1}, \ldots, \beta_{k}\right)^{T}$ and $\varphi_{k}(., \Omega)$ is the density of multivariate normal distribution with $k \times k$ correlation ma$\operatorname{trix} \Omega=\left(\rho_{i j}\right)$. Since $f_{n, m}(\mathbf{z} ; \boldsymbol{\alpha}, \boldsymbol{\beta}, \Omega)$ is a density function, thus we have $c=E_{\mathbf{X}}\left\{\left[\Phi\left(\boldsymbol{\alpha}^{T} \mathbf{X}\right)\right]^{n}\left[\Phi\left(\boldsymbol{\beta}^{T} \mathbf{X}\right)\right]^{m}\right\}$, where $\mathbf{X} \sim N_{k}(\mathbf{0}, \Omega)$. 


\section{Acknowledgment}

The authors would like to thank the two referees for their helpful comments and suggestions. Furthermore, the authors is indebted to professor A. Jamalizadeh of the Shahid Bahonar University of Kerman for their encouragement and many helpful discussions.

\section{References}

Arellano-Valle, R.B., Gomez, H.W. and Quintana, F.A. (2004). A new class of skew-normal distribution. Communication in Statistics - Theory and Methods, 33, 1465-1480.

Arnold, B.C. and Beaver, R.J. (2002). Skewed multivariate models related to hidden truncation and/or selective reporting (with discussion). Test, 11, 7-54.

Azzalini, A. (1985). A class of distributions which includes the normal ones. Scandinavian Journal of Statistics, 12, 171-178.

Azzalini, A. (1986). Further results on a class of distributions which includes the normal ones. Statistica, 46, 199-208.

Azzalini, A. and Dalla Valle, A. (1996). The multivariate skew-normal distribution. Biometrika, 83, 715-726.

Branco, M. and Dey, D. (2001). A general class of multivariate elliptical distributions. Journal of Multivariate Analysis, 79, 99-113.

Gupta, R.C. abd Gupta, R.D. (2004). Generalized skew-normal model. Test, 13, 501-524.

Henze, N.A. (1986). A probabilistic representation of the skew-normal distribution. Scandinavian Journal of Statistics, 13, 271-275.

Jamalizadeh, A., Behboodian, J. and Balakrishnan, N. (2008). A two-parameter generalized skew-normal distribution. Statistical and Probability Letters, 78, 1722-1728.

Kotz, S., Balakrishnan, N. and Johnson, N.L. (2000). Continuous Multivariate Distributions - Vol. 1, second edition. Wiley, New York.

Kotz, S. and Johnson, N. (1985). Orthant probability. Encyclopedia of statistics 6, 521-523, Wiley, New York.

Sharafi, M. and Behboodian, J. (2008). The Balakrishnan skew-normal density. Statistical Papers, 49, 769-778.

Steck, G.P. (1962). Orthant probability for the equicorrelated multivariate normal distribution. Biometrika, 49, 433-445. 
Yadegari, I., Gerami, A. and Khaledi, M.J. (2008). A generalization of the Balakrishnan skew-normal distribution. Statistical and Probability Letters, 78, 1156-1167.

\section{Wahab Bahrami}

Department of Statistics,

Faculty of Mathematics and

Computer Science,

Shahid Bahonar University of Kerman,

Kerman, Iran.

email:W.Bahrami@yahoo.com

\section{Hamzeh Agahi}

Department of Statistics,

Faculty of Mathematics and

Computer Science,

Shahid Bahonar University of Kerman,

Kerman, Iran.

\section{Hojat Rangin}

Department of Statistics,

Faculty of Mathematics and Computer Science,

Tarbiat Moalem University of Tehran,

Tehran, Iran. 